\section{Habituation or sensitization? Response to Leaton}

\author{
EDWARD J. SCHICATANO \\ Bowman Gray School of Medicine, \\ Winston-Salem, North Carolina \\ and \\ TERRY D. BLUMENTHAL \\ Wake Forest University, \\ Winston-Salem, North Carolina
}

In our recent Psychobiology article (Schicatano \& Blumenthal, 1994), we stated in the title and in our conclusion that caffeine delays habituation of the acoustic startle response. Leaton (1995), in a note in the present issue of Psychobiology, makes the valid argument that the data do not conclusively indicate whether caffeine "delayed habituation" or "enhanced sensitization." Habituation and sensitization are two theoretical processes that may be occurring concurrently to influence a response to redundant stimulation (Groves \& Thompson, 1970; Thompson \& Spencer, 1966). The data for our Figures 1 and 3 (Schicatano \& Blumenthal, 1994) demonstrate no increases in response amplitude in either the caffeine or the placebo conditions following the first trial block as would be expected from a "predominant" sensitization process. Furthermore, in the placebo condition (in both Figures 1 and 3), a significant decrease in response amplitude was observed as early as Trial Block 2 . Since we observed a standard habituation curve in our placebo condition that was modified in our caffeine condition, we concluded that the process that was disrupted was probably habituation. Notice that caffeine did not make subjects more responsive in the second trial block than in the first trial block. Also, the findings of Smith, Rafferty, Lindgren, Smith, and Nespor (1991) showed that caffeine slowed habituation of the skin conductance response, a result that does not suggest that "one might expect the drug to enhance sensitization" as Leaton suggests.

The examples used by Leaton to argue against a "habituation" explanation are based on studies that do not unequivocally show that sensitization is the predominant process occurring instead of habituation (Borszcz, Cranney, \& Leaton, 1989; Young, Helmstetter, Rabchenuk, \& Leaton, 1991). Furthermore, the sensitization findings that they describe are "controlled by associative variables" (Borszcz et al., 1989), contradicting the basic tenets of

Correspondence should be addressed to T. D. Blumenthal, Department of Psychology, Wake Forest University, Box 7778 Reynolda Station, Winston-Salem, NC 27109 (e-mail: blumen@wfu.edu). the dual-process theory, which considers sensitization to be a nonassociative form of learning.

Boelhouwer, Gregoric, van den Bosch, Schomaker, and Brunia (1982) have shown that the early Rl component of the electrically elicited startle response sensitizes with repeated presentation, whereas the late R2 component does not. This $\mathbf{R} 2$ component is analogous to what we measure with the acoustic startle response, suggesting the existence of a single fundamental process common to this component, this being habituation.

Of course, Leaton is correct in noting that it is difficult to look at a single response system and distinguish between delay of habituation and sensitization on the basis of the failure to show decreased responding. Delay of habituation requires the absence of a process that normally occurs. Sensitization in this case requires the presence of two processes, sensitization and habituation, with sensitization compensating for habituation, bringing responding back to the original level. Is it more parsimonious to assume that an expected process such as habituation is not occurring, or that two processes are occurring and are in balance?

Finally, if we define response habituation as a decrease in responding with repeated stimulus presentation, then we have response habituation in both our placebo and caffeine conditions. Since this response habituation occurs later in the session in the caffeine condition than in the placebo condition, we operationally define "delay of habituation" as response habituation that occurs later in time. This is based on the distinction made by Groves and Thompson (1970) between the hypothetical construct of "habituation" and the observation of a decrease in responding, called "response habituation." The theoretical distinction between the delay of habituation and sensitization is important, as Leaton suggests, but this distinction is not central to our conclusion.

\section{REFERENCES}

Boelhouwer, A. J. W., Gregoric, M., van den Bosch, W. E. J., Schomaker, L. R. M., \& Brunia, C. H. N. (1982). Habituation of the human blink reflex: The effect of stimulus frequency and the state of arousal. Physiological Psychology, 10, 325-330.

Borszcz, G. S., Cranney, J., \& Leaton, R. N. (1989). Influence of long-term sensitization on long-term habituation of the acoustic startle response in rats: Central gray lesions, preexposure, and extinction. Journal of Experimental Psychology: Animal Behavior Processes, 15, 54-64.

Groves, P. M., \& Thompson, R. F. (1970). Habituation: A dualprocess theory. Psychological Review, 77, 419-450.

LEaton, R. N. (1995). Comment on Schicatano and Bluemnthal (1994): Habituation or sensitization? Psychobiology, 23, 85-86.

Schicatano, E. J., \& Blumenthal, T. D. (1994). Caffeine delays habituation of the human acoustic startle reflex. Psychobiology, 22, 117-122.

Smith, B. D., Raffert y, J., Lindgren, K., Smith, D. A., \& Nespor, A. 
(1991). Effects of habitual caffeine use and acute ingestion: Testing a biobehavioral model. Physiology \& Behavior, 51, 131-137.

Thompson, R. F., \& SPEncer, W. A. (1966). Habituation: A model phenomenon for the study of neuronal substrates of behavior. Psychological Review: 73. 16-43.

Young, B. J., Helmstetter, F. J., Rabchenuk, S. A., \& Leaton, R. N.
(1991). Effects of systemic and intra-amygdaloid diazepam on longterm habituation of acoustic startle in rats. Pharmacology, Biochemistry \& Behavior, 39, 903-909.

(Manuscript received November 11, 1994; accepted for publication November 23, 1994. 